\title{
Computer aided control and monitoring of aquaculture plants
}

\author{
ERIK HANSENं
}

Keywords: Data acquisition, automatic control, monitoring, logging, trend analysis, interactive dynamic colour display, alarm.

\begin{abstract}
A computer system for control and monitoring of aquaculture plants has been developed by the Norwegian company NODEC. The system is based on a local area network which interfaces a computer system to the primary instruments located at various sites in the plant. Special software modules have been developed to handle different tasks such as data gathering, automatic control, alarming, logging, trend analysis and reporting. Special effort has been made to develop a user-friendly high level man-machine interface. A software library is available for monitoring and control of water quality, water distribution, heating systems, biomass and feeding systems.
\end{abstract}

\section{Introduction}

This paper deals with the structure and mode of operation of a system for monitoring and control of aquaculture plants. The demands on the system and the basis of these demands are dealt with, as are the structure and mode of operation of the system as such. Special emphasis is placed on the technical solution applied to building a local data network for data acquisition and control.

The system described has the designation NCS-100, recently announced by NODEC in Trondheim, Norway.

\section{Automation and monitoring procedures in aquaculture plants}

In aquaculture plants there is a need for extensive monitoring to obtain the best possible control over the different environmental parameters which are important to the production process.

Landbased plants in particular also have a number of technical installations which it is necessary to monitor and control. These circumstances have often caused aquaculture plants to be compared to the processing industry with regard to monitoring and control. It follows logically that the systems for data aquisition and control used in the processing industry are also used in such plants. An important question is whether these systems are suitable for the task they are supposed to deal with.

To answer this question it is necessary to find out which parameters we want to control and monitor and how this should be done. With this as a basis it should be

Received 15 January 1987.

This paper was presented at the IFAC Symposium on Automation and Dataprocessing in Aquaculture, Trondheim, Norway, 18-20 August 1986.

This paper is reprinted with the permission of IFAC.

$\dagger$ NODEC A/S, 7000 Trondheim, Norway. 
possible to assert what demands must be presented and how such systems should function.

\subsection{Needs for monitoring}

For aquaculture plants it is of great importance to have a system capable of gathering data on:

Environment parameters like water quality, water temperature, air pressure, wind etc.

Stock i.e. the total biomass and the number of specimens being bred.

Technical installations used in the plant.

The gathered data will serve as a basis for:

Status summary

Alarms

Reporting

Statistics

Estimates for use in production planning, complex control functions, simulations and optimizing

Control.

\subsection{Need for control}

The gathered measurement data will also make up the basis for automatic control of the various technical installations in the plant. The most important functions to control are

Energy systems (Heat pumps)

Water distribution

Water quality

Feeding systems

\subsection{Requirements for monitoring and automation systems}

In order to meet the needs for gathering data and exerting control, the following basic demands can be presented to the system:

\section{In/Out-units}

The system must have In/Out-units to be connected to all primary instruments (sensors and actuators). This includes everything from simple on/off-signals and analog sensors to intelligent measurement-stations and distributed single-loop controllers. It should be fairly easy to connect primary instruments after the system is put into operation. Each connecting point should have a low price. Transmission of data to and from the primary instruments should be quick and noise-immune. 


\section{Computer-system}

The computer system should be able to deal with the following tasks

I/O-system supervising

Regulator-functions (P, PI, and PID)

Alarm supervising

Data handling (logging, trend analysing and reporting)

Math and logic functions

Real time clock functions

\section{Operator-system}

For communication with the operator a colour-graphic VDU-terminal should be used for

Configuration and maintenance of the system

Presentation of system status through interactive dynamic colour graphics

Presentation of data trends

Manual control

Presentation of alarms and messages

Handling program system for production planning, optimizing and simulation

\section{Software}

The system must contain software for

Configuration of the system

Linearization, trend analysis and statistical processing of measurement data

P, PI and PID-regulator functions and parameter estimation

Simulation and calculation of optimal production parameters

Reporting and logging functions

User interface

\section{General demands}

User friendliness

Fail-safe (redundancy)

Flexibility (easy to expand)

Easy maintenance

Interface towards other systems

Low cost regarding purchase, installation and expansion

The requirements on monitoring and control is presented in the following figure (Fig. 1). 


\begin{tabular}{|c|c|c|}
\hline $\begin{array}{c}\text { Dota- } \\
\text { ocipuisition } \\
\text { system }\end{array}$ & $\begin{array}{l}\text { Requirements on a monitoring } \\
\text { and automation system }\end{array}$ & $\begin{array}{c}\text { PLC- } \\
\text { system }\end{array}$ \\
\hline Limited & Interactive graphic display & Limited \\
\hline No & Low-cost $1 / 0$ & Yes \\
\hline No & Regulator functions & $Y \in s$ \\
\hline Limited & Math and logic functions & Yes \\
\hline No & Alarm supervising & Yes \\
\hline No & Real time database & No \\
\hline Limited & High-level contiguration & Lımsted \\
\hline Yes & Dato handlıng and logging & No \\
\hline No & Statistical processing & tio \\
\hline No & Simulation and calculation & No \\
\hline
\end{tabular}

Figure 1. Monitoring and control requirements.

\subsection{Comparison of PLC and data acquisition systems}

Properties specific to the aquaculture plant in comparison with the processing plant are:

Complex biological and chemical processes

Long-range production planning

Slow process, difficult to get general view of the process

Complex interaction of different factors such as stock, food, energy, water quality etc

These properties lead to the following basic requirement for a monitoring and control system for aquaculture plants; it should be a diagnostic system based on historical data as much as a realtime control system.

Programmable logic control (PLC) is often used for automation purposes in the process industry. One of the main drawbacks with these systems is their lack of status-presentation and their limited calculation and logging possibilities. A PLC system gives the operator a 'snapshot' of the status in the plant.

To cope with these problems, data-acquisition systems are often used. These systems, however, do not have the required alarm, regulation and control functions. The answer to my previous question is therefore: PLC and data acquisition systems are not suitable for monitoring and control in aquaculture plants.

The ideal system is one which includes the best of the two worlds and in addition has the required calculation and presentation capabilities.

\section{Node Controlling System 100}

The NCS-100 is a distributed system for data acquisition and control, designed for use within process control and monitoring. The NCS-100 consists of the following main components: host computer, network controller, local area network (LAN) and nodes (Fig. 2). 


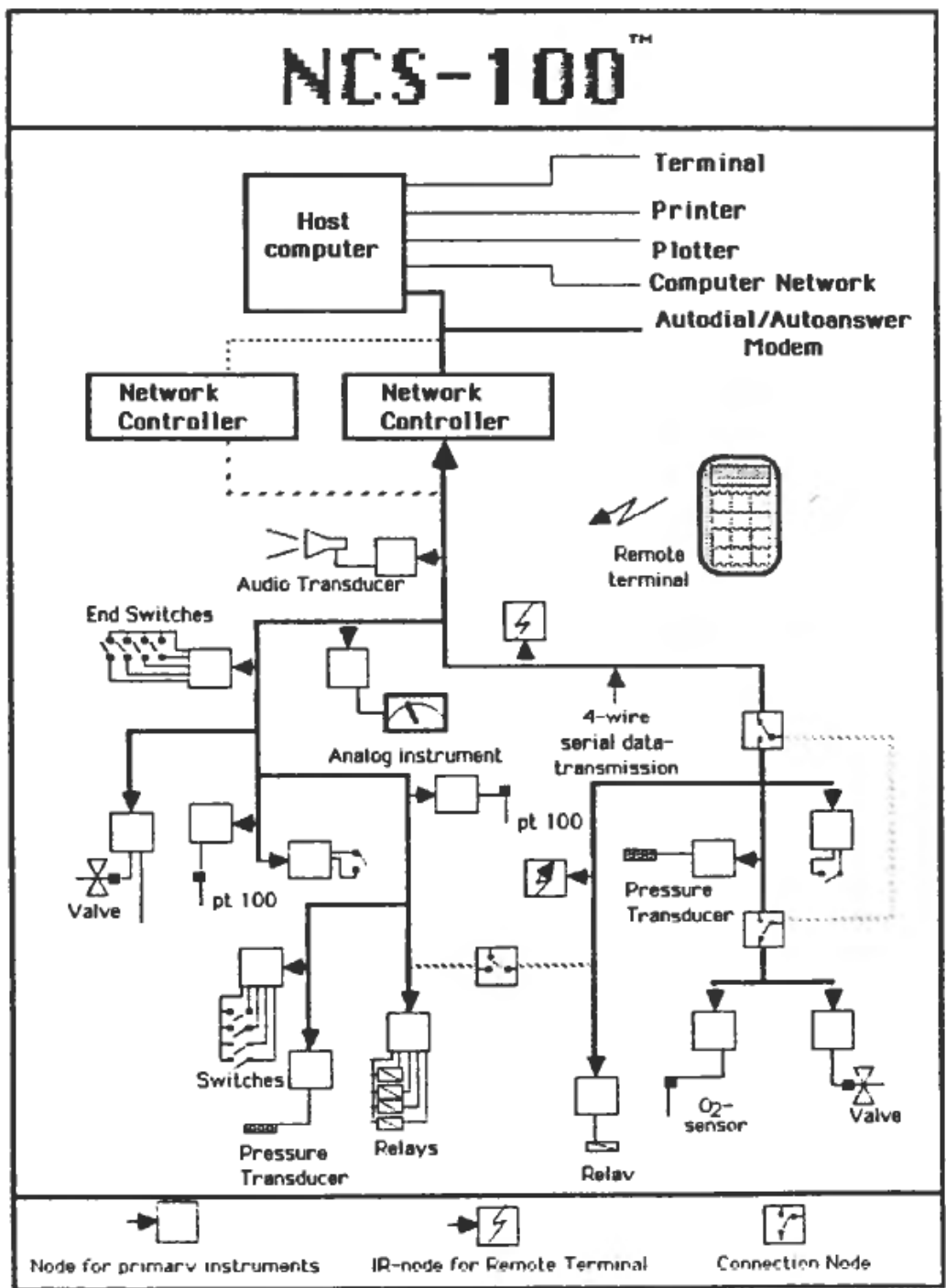

Figure 2. The NCS-100.

The system is based on an intelligent network controller which operates the local area network for data transfer to and from primary instruments. The network controller operates on a basis of programmed instructions transferred from a host mini or micro computer. The status in the network and the logged data are transferred from the network controller to the host computer. The nodes are used as interfaces between the primary instruments and the LAN. The system is based on VLSI-technique and is optimized with regards to power consumption, transfer rate and program efficiency.

\subsection{Host computer}

A mini or micro computer is used for system configuration, status information and logging. The host computer communicates with up to 16 network controllers 


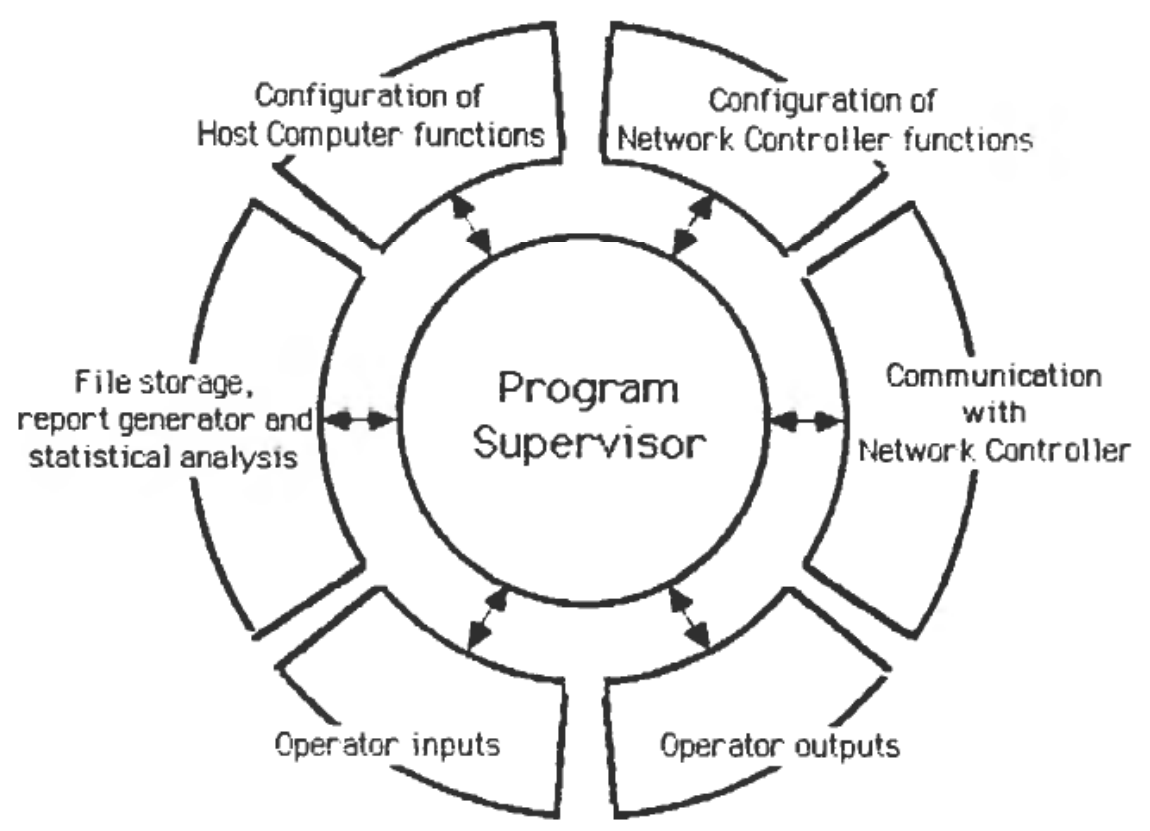

Figure 3. Interaction between host computer program routines.

via the same RS-232 or RS-422 communication channel. For remote control purposes, communication can take place via a modem.

The software in the host computer is menu-based and is adjusted to the different fields of use of the system. The software supplied has initially been adapted to the IBM-PC/XT/AT family of micro computers. The computer must be equipped with a $10 \mathrm{M}$-byte hard-disc minimum. Figure 3 shows the interaction between the program routines in the host computer. The following functions are performed by the host computer:

\section{System configuration}

When starting a system for control and monitoring, parameters for each of the nodes together with their functional relationship to each other is programmed by the operator. Different displays are used during the configuration to relate the different nodes to the functions the system is supposed to take care of.

The completed configuration is translated to special instruction sequences by the host computer, and transferred to the network controller.

Interactive in-system testing may be performed, making possible a process of stepwise refinement toward a finished, working configuration. This job is simplified by the ability to interactively implement and observe the effects of configuration changes while the system is running.

\section{System status}

For presentation of the status of the plant, data is transferred from the network controller to the host computer. Here special displays stored on hard-disc are used for presentation of trends, alarms, system condition and messages. Context-relevant 
on-line help and documentation assist the user in navigating the menu system and in handling special situations (e.g. alarms and messages) as they occur.

User input is kept simple, and extensive checking and assistance provides protection against accidental erroneous input. All messages and data may be stored on hard-disc for later report generation.

\section{Statistics}

The network controller can be programmed to log values for transmission to the host computer. These values are stored on hard-disc and are presented on printouts by means of separate statistics programs. Here the operator can choose among several ways of presentation, e.g. line charts, pie charts or bar charts.

\section{Production planning and simulation}

Process data stored on disc is used by production planning and simulation programs.

\subsection{Network controller}

The network controller's task is to control the LAN according to the instructions transferred from the host computer and changes in system status. Transmission of data to the host computer is carried out for presentation and logging purposes. The network controller is based on a 16-bit micro computer with memory and peripheral circuits. The unit contains power supply, battery backup system and interface circuits for the LAN (Fig. 4).

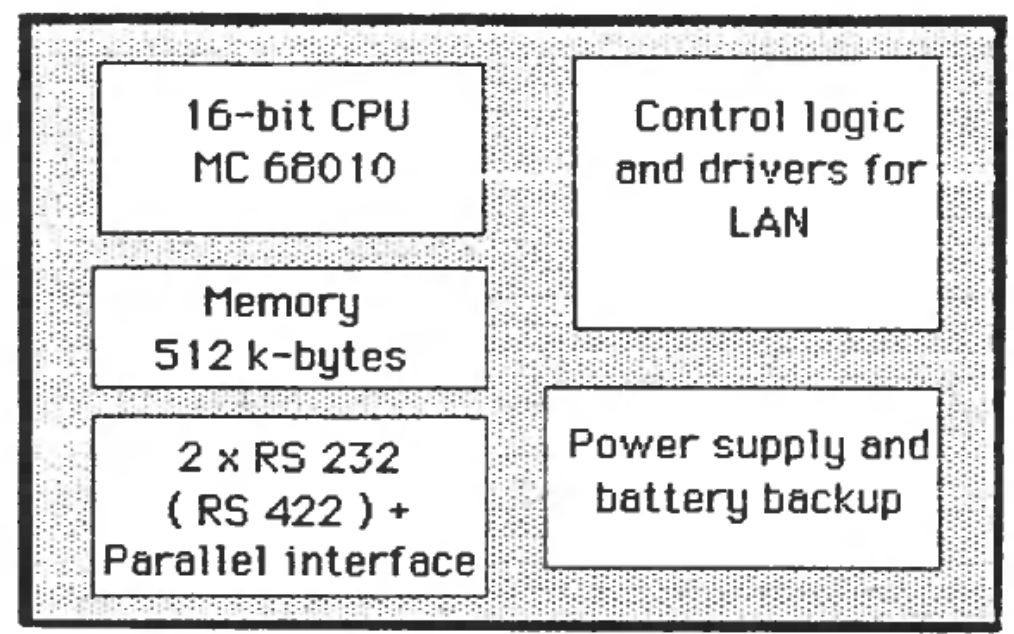

Figure 4. The network controller.

The software in the network controller consists of a real-time operating system, a dedicated control language interpreter and a number of system routines for communication and self test. Figure 5 shows the interaction between the program routines in the network controller.

For increased safety, it is possible to duplicate the network controller to achieve redundancy in the system. In such systems the host computer will, at any time, decide which controller is to control the LAN. 


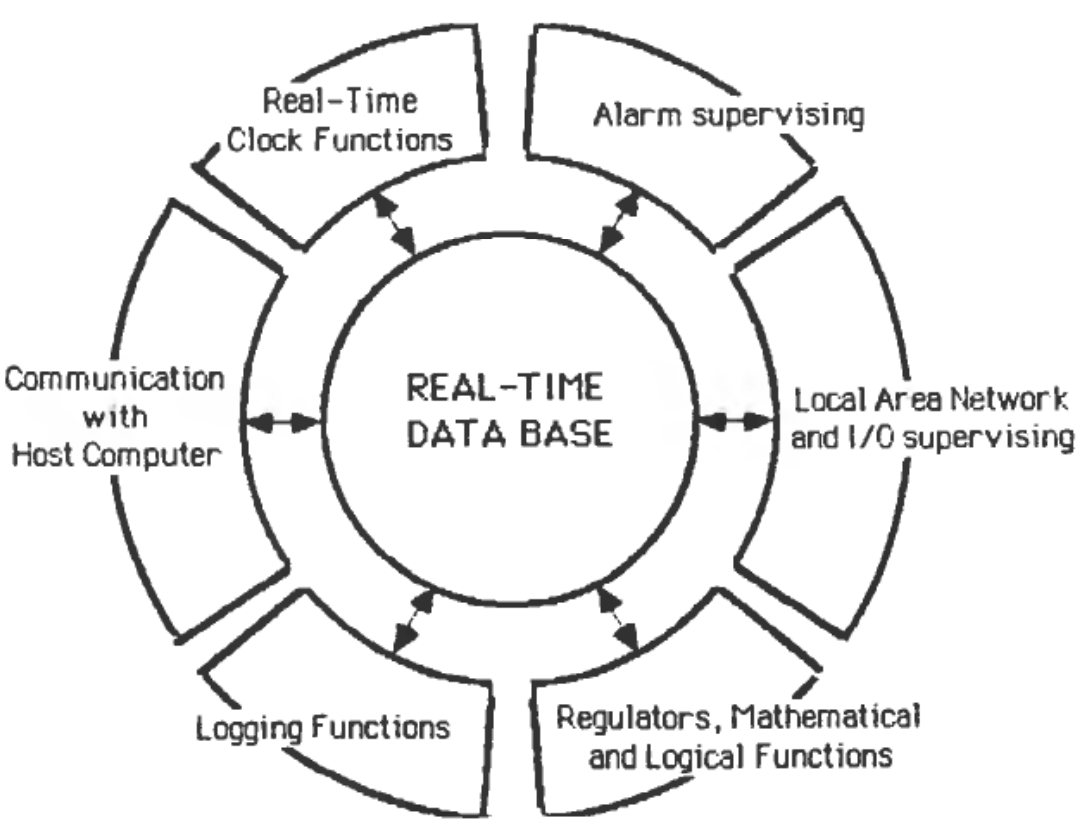

Figure 5. Program routine interactions in the network controller.

A built-in buffer is able to store messages to the host computer in case of communication break-down towards this unit. If desired an alarm can be given when the buffer is full.

The network controller is normally dependent on communication with the host computer only during system configuration and data transfer in connection with presentation of system status, logging and manual control.

\subsection{Local area network}

The network controller communicates with the different nodes via a serial busoriented local area network. The network media is a 4-wire twisted pair signal cable. Data transmission is two-way half-duplex. Each node has a 9-bit address, i.e. the network is capable of polling up to 512 nodes.

The data rate in the network is adaptive, which means that it is automatically adjusted to cable capacity and the current signal to noise ratio. The network controller can communicate with different nodes at different data rates. The data rate can vary between 150 baud and 156 kilobaud.

Manchester II biphase encoding is used for transmissions. Manchester is a selfclocking code with superior noise rejection properties. Its zero voltage-crossing during each bit time yields no dc voltage component (Fig. 6).

The data format consists of a 3-bit preamble, 9-bit address, 16-bit data and 6-bit cyclic redundancy code (Fig. 7). During the preamble sequence the node will detect the data-rate.

The structure of the LAN is bus oriented. This means that the network can be configured in an arbitrary way. For increased safety one of the peripheral branches of the network can be led back to the controller to ensure communication in case of cable failure. 


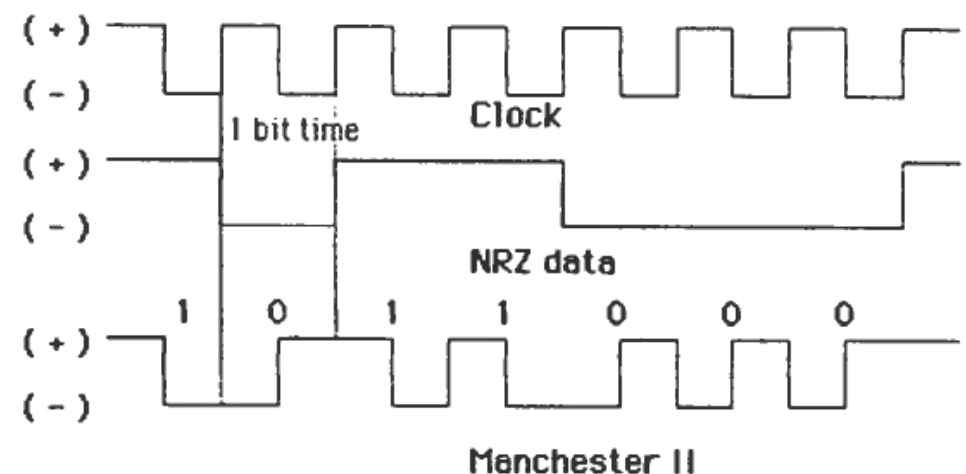

Figure 6. Manchester II biphase encoding.

\begin{tabular}{|l|l|l|l|}
\hline 3-bit preamble & 9-bit adress & 16-bit date & 6-bit CRC \\
\hline
\end{tabular}

Figure 7. The data format.

Should a cable failure occur (break or short circuit) special connection nodes will be able to reconfigure the network so that the failure can be bypassed.

The network controller can be programmed to send a test signal on the LAN. This signal is used for communication quality measurements.

Cyclic redundancy code (CRC) will detect all single and double errors, all errors consisting of an odd number of inverted bits and burst errors of length 6-bits or less.

\subsection{Nodes}

Addressable nodes are used as interface between primary instruments and the LAN. Each node consist of control circuits and interface electronics for connected instruments and LAN (Fig. 8). The control electronics are based on a CMOS VLSI-

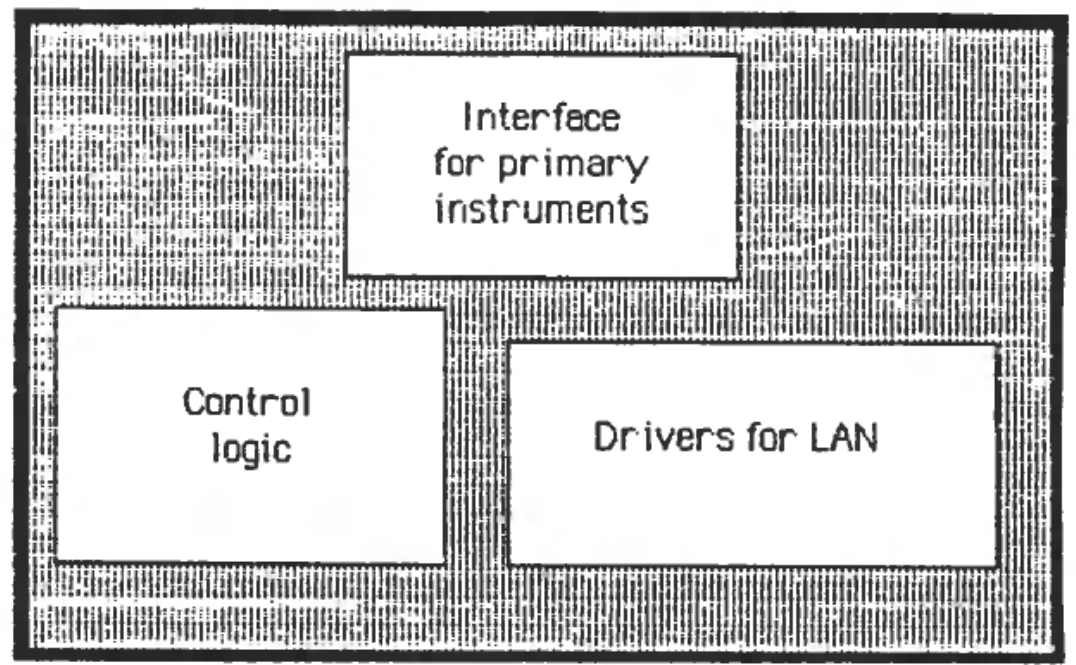

Figure 8. An addressable node. 
circuit specially designed for this purpose and produced for NODEC by the Hitachi Corp. in Japan.

The nodes are delivered in different enclosures according to specific environmental demands. The size of each node is $4 \times 6 \mathrm{~cm}$. In special cases a node can be integrated in the individual sensor. The following nodes are supplied for the system:

16 Digital inputs

16 Digital outputs

4 Analog inputs

4 Analog outputs

IR-Receiver/transmitter

Connection node

Counter node

\subsection{Portable terminal}

The system will be supplied with a portable terminal for display of system status and input of system parameters. The portable terminal will be in wireless communication with installed IR-nodes and is battery powered. Simple command sequences can be executed via programmable function keys.

\section{Typical installation}

This chapter will give an example of a typical installation of NCS-100 in an aquaculture plant. A hatchery and fry non-recirculating plant with a capacity of 1 million smolt are used in this example. The system will support the following functions:

Biomass-accounting system

Control and monitoring of

Automatic feeding

Heat pump

Water distribution

Water quality

The menu system configured on the host computer is shown in Fig. 9.

\subsection{Description of supported functions}

Biomass accounting system: This function is based on manual input of specimen and biomass for each of 150 fish rearing units. These data form the basis for growthcalculations and reports and are key data for the control of the automatic feeding system.

Automatic feeding system: 60 feeding units are controlled by solenoid-valves. Food consumption and stock for the units are monitored by load-cells. The control program acts on the basis of data from 'Touch-food sensors,' biomass accounting system, water temperature, water quality system, time, production plans and oper- 


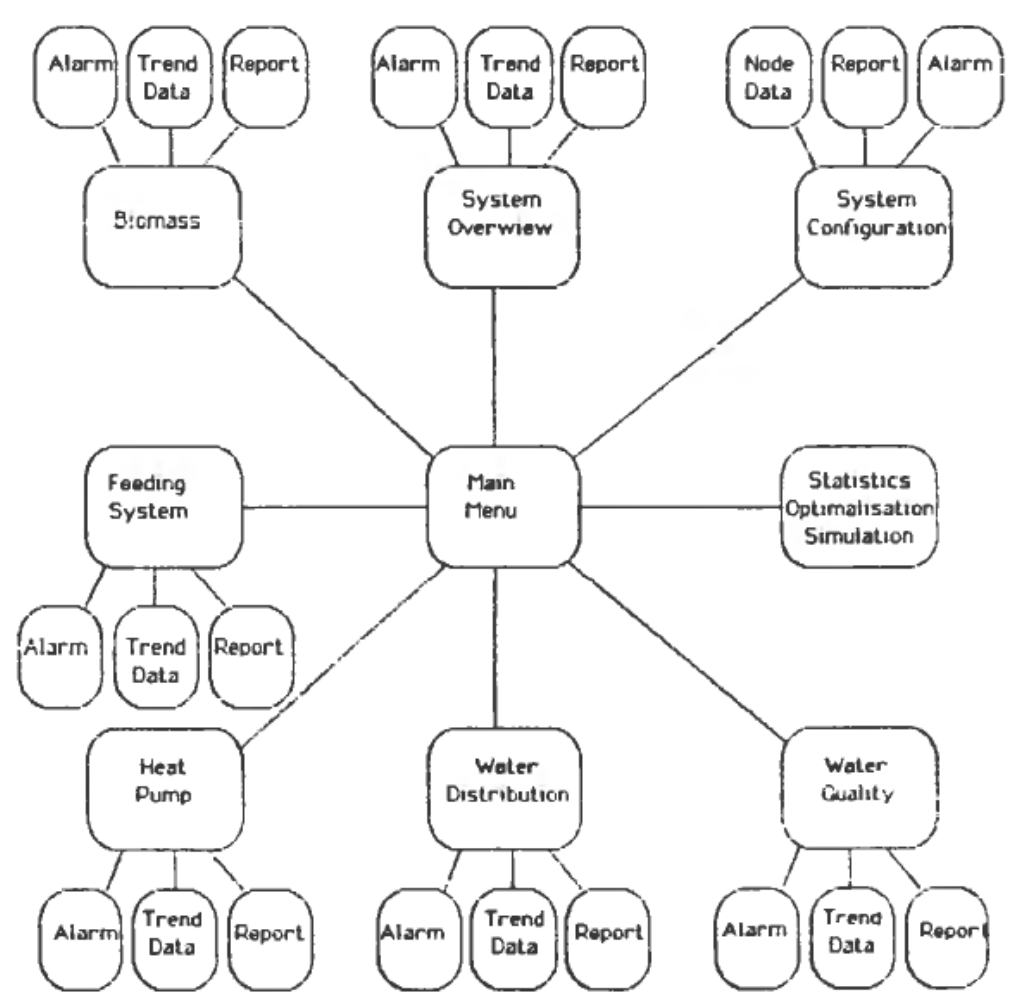

Figure 9. Host computer menu system.

ator inputs. Reports are made on food consumption, food factor and fish activity. The automatic feeding system gives alarm on malfunction of feeding units, low and high limits of food-consumption/food-factor, low and high limits of fish activity.

Heat pump system is monitored by means of 8 temperatures, 2 flow-sensors (main inlet and outlet) and energy consumption in the compressor. These parameters are logged and used for power-factor calculation and automatic control of the heat pump. Reports and alarms are made on the 8 temperatures and power-factor.

Water distribution: The water-flow in the different sections is monitored by means of 6 flow-sensors in addition to the ones used by the heat pump system. Water level in 6 reservoir-tanks is also monitored. A malfunction in the water supply will cause the system to invoke automatic recirculation by controlling valves and pumps. Alarms are made on high and low limits on flow and water levels. Reports are made on flow and water levels.

Water quality is monitored by means of 3 oxygen-sensors, $2 \mathrm{pH}$-sensors, 1 conductivity-sensor, 1 sensor for unsaturated gases in the water and 1 turbidity-sensor. The measured values are logged regularly for report generation. Alarms are given when values reach preset levels. Oxygen is added automatically when the measured value gets too low.

\subsection{Input/output requirements}

The number of $\mathrm{I} / \mathrm{O}$-connections needed for the functions described are listed in Fig. 10. 


\begin{tabular}{|c|c|c|c|c|}
\hline 1/0-Type: & $\begin{array}{c}\text { Digital } \\
\text { Input }\end{array}$ & $\begin{array}{l}\text { Digital } \\
\text { Output }\end{array}$ & $\begin{array}{c}\text { Analog } \\
\text { Inout }\end{array}$ & $\begin{array}{l}\text { Analog } \\
\text { Outout }\end{array}$ \\
\hline Feeding System : Feed Units & & 60 & & \\
\hline Touch-Food Sensors & 60 & & & \\
\hline Loed Cells & & & 60 & \\
\hline Heat Pump : Flow Sensors & & & 2 & \\
\hline Temperature sensors & & & 8 & \\
\hline Watt Meter & & & 1 & \\
\hline Compressor Control & & & & 1 \\
\hline Woter Distribution : Valves & & & & 4 \\
\hline Pumps & & 3 & & 1 \\
\hline Flow Sensors & & & 6 & \\
\hline Level Sensors & 4 & & 2 & \\
\hline Woter Quality : $\mathrm{O}_{2}$ Sensors & & & 3 & \\
\hline pH Sensors & & & 2 & \\
\hline Conductivity Sensors & & & 1 & \\
\hline Ges saturation Sensors & & & 1 & \\
\hline Solinity Sensors & & & 1 & \\
\hline Turbidity Sensors & & & 1 & \\
\hline Control of $\mathrm{O}_{2}$ & & & & 1 \\
\hline Totel : & 64 & 63 & 88 & 7 \\
\hline
\end{tabular}

Figure 10. Input/output connection requirements.

\section{REFERENCES}

INGEBRIGTSEN, O. (1982). Akvakultur, Oppdrett av Laksefisk. (NKS: Oslo), Chapters 4, 5, 14. LADUZINSKY, A. J. (1985). As serial communications buses proliferate, will standards develop? Control Engineering, October, 51-52.

MORDT, H. E. (1985). Intelligent instrumentenhet som VLSI-krets? Elektro (Oslo), September, 24-29.

NORMAK, P. E. (1985). Lokale nett for desentralisert styring. Elektro (Oslo), March, 31-32.

Pinkowitz, D. C. (1985). A standard local network for industrial use. Control Engineering, October, 56-57.

TVENNING, H. T. (1980). Fiskeoppdrett. (Aschehoug: Oslo), pp. 38-51. 